\title{
Lesion distribution characteristics of deep infiltrating endometriosis with ovarian endometrioma: An observational clinical study
}

\section{CURRENT STATUS: UNDER REVIEW}

BMC Women's Health BMC series

Hungling Kwok

the Seventh Affiliated Hospital, Sun Yat-sen University

Hongye Jiang

Sun Yat-sen University First Affiliated Hospital

Tian Li

the Seventh Affiliated Hospital, Sun Yat-sen University

Huan Yang

the seventh Affiliated Hospital, Sun Yat-sen University

Hui Fei

the Seventh Affiliated Hospital, Sun Yat-sen University

Li Cheng

the Seventh Affiliated Hospital, Sun Yat-sen University

Shuzhong Yao

Sun Yat-sen University First Affiliated Hospital

Shu-Qin Chen

Sun Yat-sen University First Affiliated Hospital

$\checkmark$ chenshuqin1021@163.comCorresponding Author

\section{DOI:}

10.21203/rs.2.19864/v1

\section{SUBJECT AREAS}

Obstetrics \& Gynecology

\section{KEYWORDS}

ovarian endometrioma, deep infiltrating endometriosis, lesion distribution characteristics 
Abstract

Purpose: To investigate the characteristics of deep infiltrating endometriosis (DIE) lesion distribution when associated with ovarian endometrioma (OEM).

Methods:The present study analyzed retrospective data obtained by the First Affiliated Hospital of Sun Yat-sen University, between June 2008 to June 2016. A total of 304 patients underwent laparoscopic surgery for complete removal of endometriosis by one experienced surgeon, and histological confirmation of OEM associated with DIE was conducted for each patient. Clinical data were recorded for each patient from medical, operative and pathological reports. Patients were then divided into two groups according to unilateral or bilateral OEM. Patients with unilateral OEM were subsequently divided into two subgroups according to OEM location (left- or right-hand side) and the diameter of the OEM ( $\leq 50$ and $>50 \mathrm{~mm}$ ). The distribution characteristics of DIE lesions were then compared between the groups.

Results:DIE lesions were widely distributed, 30 anatomical sites were involved. Patients with associated unilateral OEM ( $n=184$ patients) had a significantly increased number of DIE lesions when compared with patients with bilateral OEM ( $n=120$ patients; $2.76 \pm 1.52$ vs. $2.33 \pm 1.34 ; P=0.006$ ). Compared with bilateral OEM with DIE, there was a higher rate of intestinal (39.1\% vs. $18.3 \%$; $\mathrm{P}<0.01)$ and vaginal $(17.4 \%$ vs. $6.7 \% ; \mathrm{P}<0.01)$ infiltration by DIE lesions in unilateral OEM with DIE. The mean number of DIE lesions was not significantly correlated with the location or size of the OEM ( $2.83 \pm 1.56$ vs. $2.74 \pm 1.53 ; P=0.678$; and $2.65 \pm 1.42$ vs. $2.80 \pm 1.43 ; P=0.518$, respectively). There was no significant difference between the groups with OEM $\leq 50 \mathrm{~mm}$ and $>50 \mathrm{~mm}$.

Conclusion:Lesion distribution characteristics in women diagnosed with histologically proven OEM associated with DIE were frequently multifocal and severe.

Full Text

Due to technical limitations, full-text HTML conversion of this manuscript could not be completed. However, the manuscript can be downloaded and accessed as a PDF.

\section{Tables}

Table I Preoperative characteristics of the study population. 


\begin{tabular}{lc}
\hline Patients' characteristics $(\mathrm{N}=304)$ & Values $^{\mathrm{a}}$ \\
\hline Age(years) & $34.2 \pm 5.8($ range17-49) \\
Parity & $158(52.0 \%)$ \\
Para $\geq 1$ & $146(48.0 \%)$ \\
Nulliparous & $78(25.7 \%)$ \\
Infertility & $88(28.9 \%)$ \\
Previous surgery for OEM & $32(10.5 \%)$ \\
Presenting symptoms ${ }^{\mathrm{b}}$ & $272(89.5 \%)$ \\
No symptoms & $39(12.8 \%)$ \\
Dysmenorrhea & $17(5.6 \%)$ \\
Dyspareunia & \\
Chronic pelvic pain & $73(24.0 \%)$ \\
& \\
Anus bulge & $13(4.3 \%)$ \\
& $6(2.0 \%)$ \\
Difficulty in defecation & $11(3.6 \%)$ \\
Diarrhea & $8(2.6 \%)$ \\
Defecation pain & $9(3.0 \%)$ \\
Hematochezia & $2(0.7 \%)$ \\
Urinary frequency and urgency & $26(8.6 \%)$ \\
Haematuria & \\
Endronephrosis & \\
Left & $98(32.2 \%)$ \\
Right & $86(28.3 \%)$ \\
Bilateral & $120(39.5 \%)$ \\
Endometrioma laterality & $2.60 \pm 1.5(\mathrm{range}-9)$ \\
Number of DIE lesions & \\
\hline
\end{tabular}

OEM, ovarian endometrioma; DIE, deep infiltrating endometriosis.

${ }^{a}$ Values are shown as mean \pm standard deviation or $\mathrm{N}(\%)$.

${ }^{b}$ Different symptoms can be associated in the same patient.

Table II The prevalence of anatomical distribution of DIE lesions. ( $n=304$ patients) 


\begin{tabular}{ll}
\hline Main lesion $^{\mathrm{a}}$ & Number of patients $(\%)^{\mathrm{b}}$ \\
\hline Uterosacral ligament & $245(80.6)$ \\
Left & 57 \\
Right & 53 \\
Bilateral & 135 \\
Rectum & $93(30.6)$ \\
Ureter & $47(15.5)$ \\
& \\
Left & 22 \\
Right & 18 \\
& \\
Bilateral & 7 \\
Vaginal & $40(13.2)$ \\
Posterior fornix & $33(10.9)$ \\
Fallopian tube & $29(9.54)$ \\
Reft & 14 \\
Right & 4 \\
Vateral & 13 \\
Rectoval rectum & $21(6.91)$ \\
Bladder & $9(2.96)$ \\
\hline
\end{tabular}

DIE, deep infiltrating endometriosis.

according to the location of the lesion recorded during the operation.

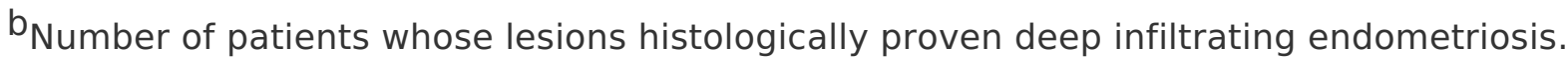

Table III Characteristics of deeply infiltrating endometriosis lesion distribution associated ovarian endometrioma. 


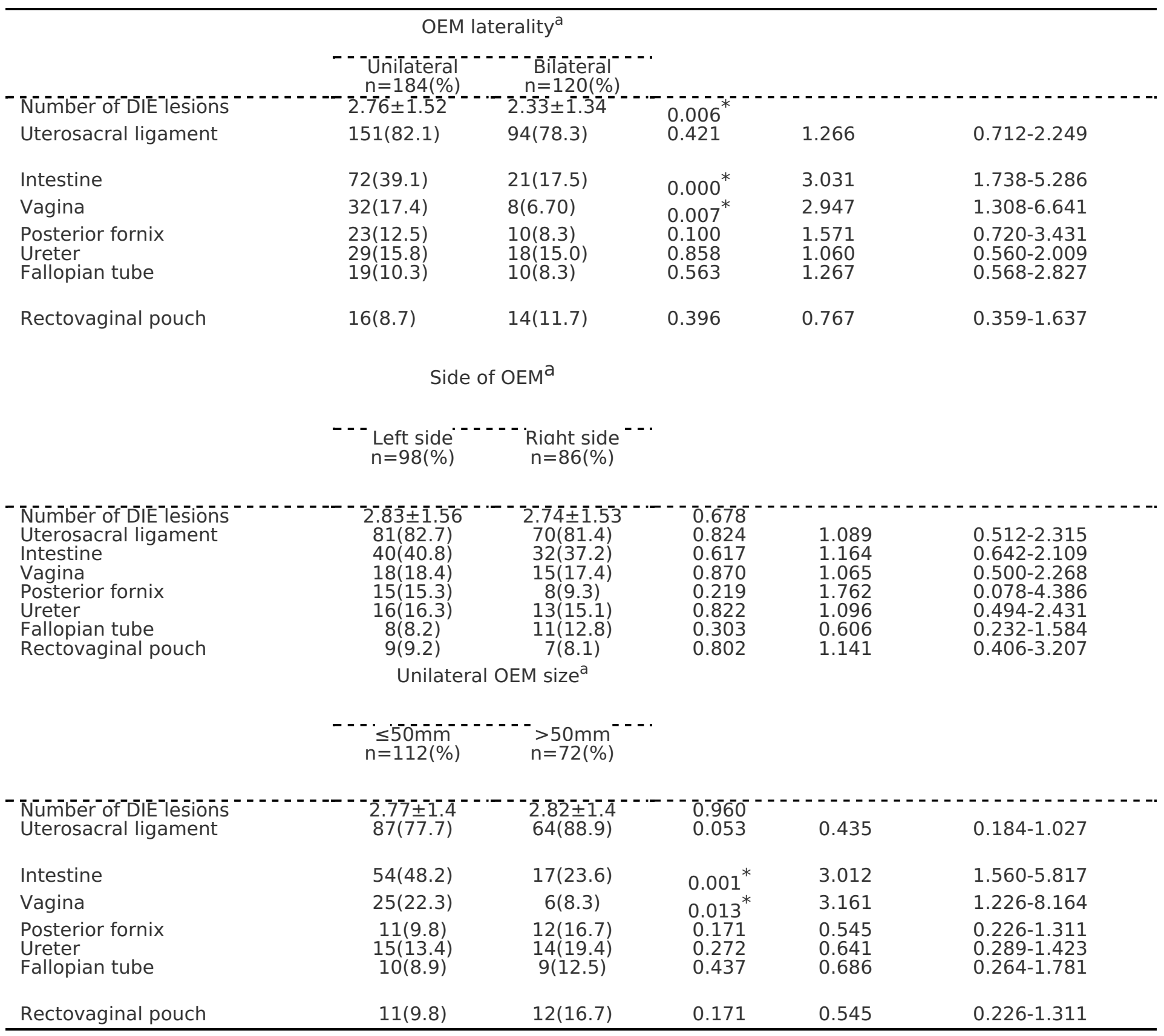

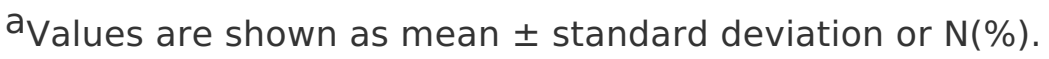

*Statistically significant 